\title{
Development of an Algorithm for Determining the Aircraft Pushback Sequence
}

\author{
Anna Kwasiborska \\ Warsaw University of Technology, Koszykowa 75 street, Warsaw 00-662, Poland, \\ e-mail anna.kwasiborska@pw.edu.pl
}

\begin{abstract}
The air transport crisis, caused by the Covid Pandemic, not only forced the limiting of air traffic operations, but also, the verification existing air traffic forecasts. This time can be used for an in-depth look at the existing procedures at the airport, namely the procedures for performing aircraft take-off operations. After the renewed increase in air traffic, the problem of proper positioning of aircraft for take-off will reappear. The capacity problem at airports will return as air traffic increases. Capacity is a parameter that determines the traffic capacity of an airport, expressed as the theoretical maximum number of air operations that the airport is able to handle per unit of time. This parameter may also result from the seasonality of air traffic both throughout the year and changes over single days or even hours. Achieving a certain capacity may require the expansion of airport infrastructure or more efficient management of airport operations. An undesirable effect of increasing air traffic are delays related to airport capacity. The existing queue of aircraft waiting for take-off operations at the runway threshold, could be due to poor management and queuing of aircraft or insufficient airport infrastructure. It is possible to eliminate the queue phenomenon, at the runway threshold, through better sequencing of aircraft push-outs. This makes it possible to determine the order of the take-off of aircraft, already at the stage of push-out. The aim of the article is to present the possibility of a more effective use of the aircraft pushback sequence, increasing the capacity of the airport maneuvering area. The proposed algorithm sequencing of aircraft to taxi in order to minimize delays associated with deviations from the flight plan, the taxiway of the stand to the runway threshold, thereby reducing emissions into the atmosphere. The algorithm is based on the A-CDM project, which unites airports to exchange information necessary to better use the capacity of these airports.
\end{abstract}

Keywords: Sequencing aircraft; capacity; taxiing; modelling

\section{Introduction}

At the beginning of the $21^{\text {st }}$ Century, in order to address the widespread problem of airport congestion, projects were created to improve airport and airspace capacity. The main reason for the changes was technical progress, as the use of 
new technologies has improved the exchange of information between important operational units. One of the main projects that aims to increase air traffic capacity is SESAR [1].

There are also other projects such as A-CDM [2] [3] and AMAN/DMAN [4] [5]. The projects support the operational work of units (ATC, air carriers, etc.) suggesting concrete real-time solutions to the problems. Unfortunately, the implementation of these projects is very time consuming and very expensive, so not all airports have fully implemented systems based on A-CDM and AMAN/DMAN projects [6]. There are airports that still face capacity problems such as: take-off queue, deicing station queue, congested aprons and lack of smooth traffic [7].

A tool for the air traffic controller to exercise safety oversight over air traffic is to impose appropriate separations between other aircraft or obstacles. Separation is the determination of the minimum value of the distance from an obstacle (or other aircraft) which guarantees the safe execution of operations [8]. Separations can be expressed in terms of distance or time. They provide protection against collision, too close a flight or flying into the turbulence area of the aerodynamic trace, which is a very dangerous phenomenon [9]. Air traffic management is based on procedures and, in some areas, on individual assessment by the controller. This management method is good for small air traffic, while in case of increased traffic, it significantly reduces capacity. The key moment to improve capacity is when an aircraft enters the traffic flow [10]. Such a flow can be identified when formulating a queue of aircraft to take off [5]. This is the moment when an aircraft is pushed back to the taxiway [11]. This operation is crucial because it can directly affect the movement of other traffic participants, causing other traffic participants to stop or be forced to change their taxiing route on the maneuvering area.

\section{Pushback of Aircraft}

Pushback of aircraft is done, only with the consent of the airport controller [9]. An aircraft declares its readiness for the pushback maneuver, and the controller analyzes visually or on the airport radar indicator the current traffic situation, and gives consent or delays the consent for the pushback. The aircraft must confirm the release of the brakes before the pushback begins. A tractor starts pushing back, by directing the aircraft to a given taxiway and positioning it in the correct direction and exactly in the axis of the taxiway. When the aircraft is in the taxiway axis, after reporting the pushback completion, it must report the brakes on and wait for the tractor to leave. To make sure that the tractor is actually disconnected, it needs to be informed both by voice and visually, because the pilot has a limited field of vision. Then the pilot asks for permission to start engines and taxiing. It is also possible that the aircraft cannot be taxied with the engines on, then the towing 
procedure takes place. The air traffic controller gives permission for towing to a suitable taxiway and to a predetermined point, where the aircraft can start engines and taxi to the runway [12].

The pushback procedure does not provide an aircraft pushback sequence optimal for the capacity of the maneuvering area, which in some situations has a significant impact on the fluidity of the traffic flow of aircraft taxiing on the taxiway to which the aircraft is pushed back [22]. The procedures do not provide for the impact of current traffic on the aircraft pushback sequences, which are subject to interpretation by the air traffic controller. Applying the order of aircraft pushback in heavy air traffic for most taxiing aircraft, it would force unnecessary waiting on the taxiway when another aircraft is pushed back.

Sequencing the aircraft to perform the take-off operation is based on the proper aircraft pushback sequence so as not to create a queue before the runway in use [13]. When the updated Target Of Block Time (TOBT) provided by the ground crew or aircraft operator to Air Traffic Control is inconsistent, warning signals are sent out to warn of the situation [14]. The Target Take-off Time (TTOT) value is calculated by VTT (Variable Taxi Time) in combination with additional times, e.g. deicing time, if this process is intended for a specific flight. Actual Start-Up Approval Time (ASAT) and TTOT are transferred via the A-CDM Information Sharing Platform (ACISP) to A-CDM partners, in particular TTOT is sent to network operators for updating [15]. Selection is a process that the ATC performs, taking into account the various aspects resulting from the expected and current traffic. ATC controllers, when making the pre-selection, must have access to the TOBT list of different aircraft, taking also into account the operational situation, and establish the ASAT either equal to or later than their TOBT. The pre-selection is then optimized by predetermined restrictions:

- $\quad$ Resulting from the Calculated Take-Off Time (CTOT) regulations

- $\quad$ The need to maximize air traffic (as many operations/h as possible)

- Ground traffic interaction (e.g. shifting of parking spaces)

For the calculated ASAT, there must be a TTOT and the necessary information such as: Default runway, Standard Instrument Departure (SID) and the size of the separation with arriving aircraft. For airports with intersecting runways and mixed take-off and landing operations there is a possibility of interference between these operations. The phenomenon occurs when the TTOT is the same as the Target Landing Time (TLDT). Therefore, the release of ASAT and consequently also TTOT takes place after the analysis of all possible loads that cause the delay. It is also possible to interfere in the selection of aircraft on a priority basis. This phenomenon most often occurs when we deal with many aircraft of one carrier, where already at the stage of TOBT determination similar values are obtained. Then, through an integrated information exchange system, information is sent to Air Traffic Management from the aircraft operator about its preferences. 
There is a regulation that refers to and prevents the sending of appropriate messages from operators to the ATC, depriving of useless information. When there is a different situation with two aircraft of different carriers, there is also an approximate TOBT. Sorting consists in assigning one of the aircraft a delay by agreement with the local target tasks and partners in A-CDM [2]. Pushback takes place when TSAT is approved.

\subsection{Aircraft Pushback Sequence Algorithm}

At airports with heavy air traffic, the phenomenon of queuing for take-off may occur [16]. It is caused by imperfect air traffic management. The factors causing this phenomenon may be flight procedures, flight schedules, weather factor or safety related random events. Procedures that do not take into account the possibility of interference in capacity management make it likely that the process will be disturbed at high traffic volumes. The procedures that particularly interfere with the possibility of a queue are:

- Pushback procedure

- Taxiing procedure from and to the parking position

- Effects of a queue:

- Increase in exhaust emissions

- Delays

- Increase in fuel consumption by the aircraft

Elimination of the phenomenon of queuing at the threshold of the runway is possible with skillful management of airport traffic through better selection of taxiways (as short as possible) and appropriate selection of the order and time of aircraft pushback. By guaranteeing the smooth movement of an aircraft from the moment of pushback to take-off, without unnecessary delays or waiting, it is possible to reduce exhaust emissions and fuel consumption of the aircraft. The analysis was based on the following assumptions:

1. Aircraft separations are fixed for a given type of operation, regardless of the aircraft type.

2. Aircraft calls are sorted per the earliest TOBT, TLDT up to the latest.

3. The time from touchdown to exit from the appropriate runway is fixed for each type of aircraft.

4. Aircraft landings are made on only one of the runways. 
The parking spaces are connected to the runway by a network of taxiways. A graph is given describing the network of air connections between the characteristic points of the airport.

$G_{j}=\left\langle X, Z P X_{j}, F X_{j}\right\rangle$

where:

$X=\left\{x_{1}, x_{2}, x_{3}, x_{4}, \ldots, x_{a} \ldots, x_{b}, \ldots, x_{p}, \ldots, x_{k}, \ldots, x_{i}\right\}$ - a set of characteristic points at the airport containing parking spaces, taxiway intersections, take-off points and points of touchdown and runway exit,

$Z P X_{j}=\left\{z p x_{j}=\left(x_{p}, x_{k}\right)\right\} \subset X \times X-$ a set of connections between points characteristic for aircraft of type $J$,

$F X j$ - specified task function: $F X_{j}: X \times Z P X_{j} \times X \rightarrow\{0,1\}$

$\forall x_{p}, x_{k} \in X, \forall z p x_{j} \in Z P X_{j} \Rightarrow F X\left(x_{p}, z p x_{j}, x_{k}\right)=\left\{\begin{array}{l}1 \text { - if exist } z p x_{j} \text { from } x_{p} \text { to } x_{k} \\ 0 \text { - otherwise }\end{array}\right.$

For each branches $z p x_{j}=\left(x_{p}, x_{k}\right)$ is defined function:

$\operatorname{Dis}\left(x_{a}, x_{b}\right)=\left\{\begin{array}{l}+R-\text { if } f x_{j}\left(x_{a,} z p x_{j}, x_{b}\right)=1 \\ 0-\text { otherwise }\end{array}\right.$

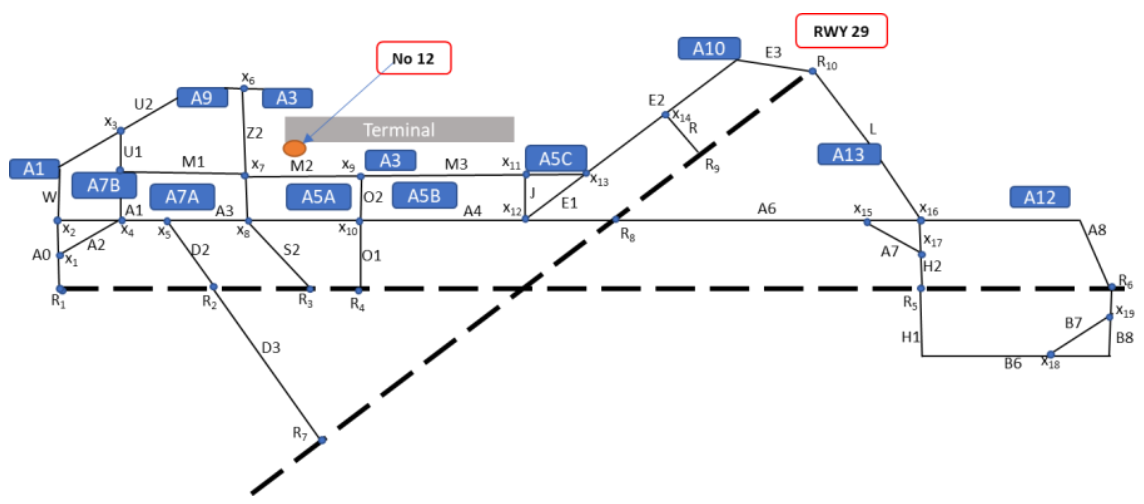

Figure 1

Scheme of structure at the airport

Diagram (Fig. 1) shows a graph created on a model airport from xi characteristic points and the possibilities of connections between them (arcs). The network of elementary operations of airport traffic is a standard network for the problem of determining extreme routes in directed networks. To determine the straight route of minimal $\mu_{\min }\left(x_{p}, x_{k}\right)$ we define: let the set $D$ of straight routes $\mu$ in the network $S_{j}=\left\langle G_{j}, \varnothing,\left\{l_{j}(u)\right\}\right\rangle$ define the function whose $F(\mu)$ values are determined by the characteristics of $f x_{j}(u)$ branches $u$ of route $\mu_{j}\left(x_{p}, x_{k}\right)$. With $D_{j}\left(x_{p}, x_{k}\right)$ we mark the set of straight routes $\mu_{j}\left(x_{p}, x_{k}\right)$ connecting vertex $x$ with vertex $x$. 
The minimum route of $\mu_{\min }\left(x_{p}, x_{k}\right)$ is one that

$F\left(\mu_{\min }{ }^{j}\left(x_{p}, x_{k}\right)=\min F\left(\mu_{j}\left(x_{p}, x_{k}\right)\right) S=\left\langle G, \emptyset,\left\{l_{j}(u)\right\}\right\rangle\right.$

where:

$G$ - graph,

$l_{j}(u)$ - actual function defined on the arc set.

Aircraft wishing to perform the take-off operation must provide the information necessary to choose the optimal taxiway. The calls are arranged from the earliest TOBT, TLD to the latest. The aircraft's call for take-off is as follows:

$Z_{s}=\left\langle x_{p}, r, T O B T, x_{k}, b\right\rangle$

where:

$x_{p}-$ starting point, parking point,

$r$ - type of aircraft,

TOBT - target time to leave the block,

$x_{k}$ - end point, starting point on the runway,

$b$ - runway intended for the take-off operation.

A priority is assigned to aircraft call so that the priority of the aircraft with earlier readiness to leave the block TOBT has a lower priority factor.

$\mathrm{TOBT}_{\mathrm{s}}<\mathrm{TOBT}_{\mathrm{s}+1} \Rightarrow p_{s}<p_{s+1}$

where:

$\mathrm{TOBT}_{\mathrm{s}, p_{s}} \in Z_{s}, \mathrm{TOBT}_{\mathrm{s}+1}, p_{s+1} \in Z_{s+1}$

Each aircraft call is also increased by the shortest taxiway it will take from the $x_{p}$ parking position to the $x_{k}$ runway point.

$D_{s}\left(x_{p}, x_{k}\right)=\mu_{\text {min }}^{j}\left(x_{p}, x_{k}\right)$ dla $r_{s}=j, r_{s} \in Z_{s}$

The same procedure is performed for reported landings.

$D_{l}\left(x_{p}, x_{k}\right)=\mu_{\text {min }}{ }^{j}\left(x_{p}, x_{k}\right)$ dla $r_{l}=j, \quad r_{l} \in Z_{l}$

Then the TTOT is calculated for the taking off aircraft

$\mathrm{TOTT}_{\mathrm{s}}=\mathrm{TOBT}_{\mathrm{s}}+\frac{\sum_{D_{s}\left(x_{p}, x_{k}\right)} \operatorname{dis}\left(x_{a}, x_{b}\right)}{V_{k}}$, for

$\operatorname{dis}\left(x_{a}, x_{b}\right) \in D_{s}\left(x_{p}, x_{k}\right)$

where:

$\operatorname{dis}\left(x_{a}, x_{b}\right)$ - distance between points $x_{a}$ and $x_{b}$ 
and TOBT

$\mathrm{TOBT}_{1}=\mathrm{TLDT}_{1}+\frac{\sum D_{l}\left(x_{p}, x_{k}\right) \operatorname{dis}\left(x_{\boldsymbol{a}}, x_{\boldsymbol{b}}\right)}{V_{k}}$

\subsection{Aircraft Taxiing}

The captain of the aircraft that is approaching the landing receives instructions from the air traffic controller about the runway in use and the taxiing route to the parking position. The aircraft may ask for "Follow Me", service assistance, during taxiing for smooth docking to a parking position. In a situation of increased air traffic, the captain is asked to leave the runway as soon as possible to allow the next air operation [17].

An aircraft ready to start taxiing notifies the air traffic controller and waits for a taxi permit. The aircraft captain receives a complete taxiing instruction containing guidelines on taxiways and possible collisions with other traffic participants on the maneuvering area. When the path designated by the traffic controller leads through the runway (intersection), the controller must give prior consent to taxiing through the intersection or provide instructions to wait [9].

The aircraft may only taxi on the runway in use with the consent of the air traffic controller. Such an operation is performed in order to speed up the air operation. In the case of operations where visual control of the air traffic controller over the aircraft is not possible, the captain of the aircraft must inform the controller of his characteristic positions.

Special procedures may occur at airports, which are related to infrastructure restrictions on aprons and taxiways.

Taxiing is one of the most important stages of designing and implementing the departure system at a given airport. The taxi procedure is initiated when the aircraft is pushed back to the taxiway, the engine(s) are turned on and the captain of the aircraft receives permission to taxi [18]. The end of taxiing time is considered to be the moment when the aircraft takes its position on the runway to perform the take-off operation. Taxiing time is determined by three main unknowns, that is: aircraft type, aircraft weight, taxiway (defined as start point, intermediate vortices and end point) [12].

The type of aircraft (large, medium, small) affects the taxiing speed and the choice of taxiways (restrictions related to the width of the main landing gear, wingspan, etc.). The weight of the aircraft mainly influences the taxiing speed, while the taxiway influences the distance traveled by the aircraft. 
Table 1

Values of taxiing times in example period at the Chopin Airport

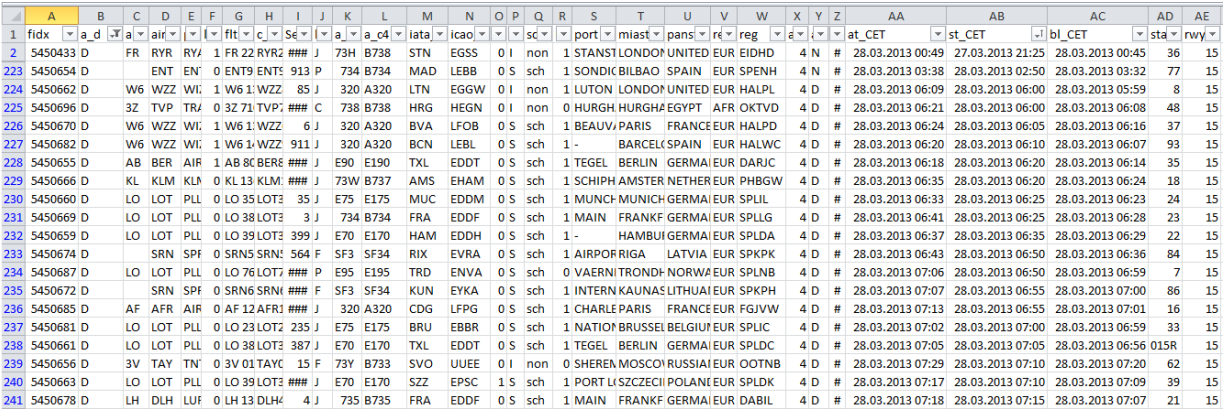

The allocation of the taxiway may be related to indirect factors resulting from congestion, deicing, the location of the parking position and starting point on the runway [9]. Identification of taxiing times is necessary to analyze the impact of infrastructure on the efficiency of operations.

Table 1 shows examples of data allowing to identify taxiways at a given airport. The data included information on the take-off point, runway in use, aircraft type, as well as the times of leaving the block, aircraft take-off and STOT scheduled take-off times.

Calculation of the actual taxi time $T_{t x}$ is the difference between the current moment of take-off and the moment the aircraft leaves the block.

$T_{t x}=\mathrm{ATOT}-\mathrm{AOBT}$

Theoretical taxiing time $T_{e o}$ can be obtained by assuming average speeds for the appropriate aircraft type $V_{k}=\{\mathrm{V} 1-24 \mathrm{~km} / \mathrm{h}, \mathrm{V} 2-28 \mathrm{~km} / \mathrm{h}, \mathrm{V} 3-32 \mathrm{~km} / \mathrm{h}\}$ over the distance between $x_{p}$ and $x_{k}$.

$T_{e o}=\frac{\operatorname{Dis}\left(X_{p}, X_{K}\right)}{V_{k}}$

For the analysis of individual taxiing times of an aircraft, the condition was assumed that the route the aircraft taxied was the shortest possible [23]. Thanks to this assumption, having at our disposal only the start and end point of the route taken by the aircraft (considering the infrastructure network is available), we can calculate the length of the route taken by the aircraft [19].

For each type of aircraft (large, medium, small) the taxiing speed has been assigned, which is necessary to calculate the theoretical taxiing time. The taxiing speed of an aircraft on the taxiway is not defined (in value) by any procedure (there is a possibility to impose restrictions by the airport traffic controller) [24]. 
Table 2

The results from at the Chopin Airport

\begin{tabular}{|c|c|c|c|c|c|c|c|c|}
\hline Delay between plan and leave apron [min] & Delay with the schedule & Taxiing time & Caterogy & Speed & axiing & Save time & Differences & $\%$ longer \\
\hline 7 & 22 & 15 & średni & 32 & 5,25 & 6 & 9 & 150 \\
\hline 1 & 11 & 10 & średni & 32 & 2,4375 & 3 & 7 & 233,3333 \\
\hline 24 & 38 & 14 & średni & 32 & 5,625 & 6 & 8 & 133,3333 \\
\hline 1 & 9 & 8 & średni & 32 & 2,71875 & 3 & 5 & 166,6667 \\
\hline 5 & 15 & 10 & mały & 24 & 6,5 & 7 & 3 & 42,85714 \\
\hline 15 & 25 & 9 & średni & 32 & 3,9375 & 4 & 5 & 125 \\
\hline 9 & 14 & 4 & średni & 32 & 2,625 & 3 & 1 & 33,33333 \\
\hline 9 & 18 & 9 & średni & 32 & 2,90625 & 3 & 6 & 200 \\
\hline 19 & 35 & 15 & średni & 32 & 3,28125 & 4 & 11 & 275 \\
\hline 10 & 19 & 8 & średni & 32 & 2,8125 & 3 & 5 & 166,6667 \\
\hline 5 & 15 & 9 & średni & 32 & 2,71875 & 3 & 6 & 200 \\
\hline 10 & 19 & 9 & średni & 32 & 3 & 3 & 6 & 200 \\
\hline 8 & 19 & 11 & średni & 32 & 4,03125 & 5 & 6 & 120 \\
\hline 11 & 23 & 11 & średni & 32 & 2,625 & 3 & 8 & 266,6667 \\
\hline 40 & 53 & 13 & Duży & 32 & 6,9375 & 7 & 6 & 85,71429 \\
\hline 1 & 13 & 11 & średni & 32 & 2,71875 & 3 & 8 & 266,6667 \\
\hline-2 & 12 & 14 & średni & 32 & 3,28125 & 4 & 10 & 250 \\
\hline 10 & 17 & 7 & średni & 32 & 2,8125 & 3 & 4 & 133,3333 \\
\hline 13 & 20 & 7 & średni & 32 & 3 & 3 & 4 & 133,3333 \\
\hline & 20,89473684 & & & & & & 6,210526 & 167,4687 \\
\hline \multirow{2}{*}{ Avarage time leaving apron } & {$[\mathrm{min}]$} & [\%] & & & & & & \\
\hline & 10,31578947 & 62,42038217 & & & & & & \\
\hline Differences of taxi time & 6,210526316 & 37,57961783 & & & & & & \\
\hline
\end{tabular}

The results obtained (Table 2) indicate that aircraft traffic on taxiways was disturbed by the influence of other aircraft. The actual taxiing time was significantly different from the theoretical one. The calculated values also show the effect of the taxiing time on the deviation of the current start time from the scheduled start time.

Two reasons for the observed delays can be distinguished. The main reason, with a $62 \%$ degree of delay to total delay, is that the aircraft leaves the block too late, which may be due to long passenger service or aircraft maintenance.

The delay associated with taxiing an aircraft on the maneuver area is $38 \%$ of the total delay. This is a very important conclusion, because it proves that not only the area of aircraft service after leaving the block (choice of routes, procedures, etc.) should be optimized, but also the areas related to passenger service (boarding method) or aircraft maintenance (earlier refueling, larger reserve of maintenance equipment, etc.) [20].

In order to better illustrate the problem of taxiing times, the discrepancies with other aircraft have been distinguished from the calculated taxiing times which can be directly compared. The comparison is only possible if the parking spaces are located a short distance from each other and the taxiing was to the same runway.

There are many methods to solve the problem of the shortest route between vortices in a directed graph. The most popular algorithms for determining minimum routes include the Bellman Ford, Dijkstra or dynamic programming algorithms [25]. The Bellman Ford algorithm, unlike the Dijkstra algorithm, allows to determine the route in the directed graph when the arc weights in the graph are negative [26]. Both algorithms are similar in terms of procedure, simple 
to implement, but unfortunately time-consuming because they determine the shortest routes from the starting vortex to all other ones. The algorithms using dynamic programming are based on reasonable decision making that gives the best possible results. The algorithms are based on one-off decision making, selection of the shortest route from among the permitted routes (meeting the task requirements, but not the specified function - minimum route).

Analyzing the route of aircraft movement from parking position no. 12 to runway RWY29 (Fig. 1), it should be pointed out that this position was usually used by wide-body aircraft. Based on the taxiway diagram and selected taxiway characteristic points, a directed graph was created (Fig. 1).

Dijkstra's algorithm is to create two sets of vortices $P$ and $K$. The sum of these sets at each stage of the algorithm must be equal to the main set of vortices of graph $V$. The $P$ set is initially empty $K=\varnothing$ and the $P$ set contains all vortices $P=V$. Then, tables of all vortices in the graph are created from $d(v)$ - the length between the vortex and the starting point, and $p(v)$ of the predecessor on the route [21].

The algorithm consists in attaching to the $\mathrm{K}$ set at the beginning of the initial vortex, and in subsequent iterations, successors/neighbors of the last added vortex from the $\mathrm{P}$ set, bearing in mind that the sum of these sets must be $V$.

The $\mathrm{P}$ set is searched for neighbors of a newly added vortex and dependency is examined:

$d\left(p_{i}\right)>d\left(k_{j}\right)+d\left(p_{i}, k_{j}\right)$

If the condition is met, the table is filled in:

$d\left(v=p_{i}\right)=d\left(k_{j}\right)+d\left(p_{i}, k_{j}\right)$ and $p\left(v=p_{i}\right)=k_{j}$

where:

$d\left(p_{i}\right)$ - length to point $p_{j}$, vortex $\mathrm{p}_{\mathrm{i}}$ belongs to set $P$,

$d\left(k_{j}\right)$ - length to point $k_{j}$, vortex $\mathrm{k}_{\mathrm{j}}$ belongs to set $K$,

$d\left(p_{i}, k_{j}\right)$ - value on $\operatorname{arc}\left(p_{i}, k_{j}\right)$.

Table 3

The shortest routes at the Chopin Airport

\begin{tabular}{|c|c|c|c|c|c|c|c|c|c|c|}
\hline$V$ & 1 & 2 & 3 & 4 & 5 & 6 & 7 & 8 & 9 & 10 \\
\hline$d(v)$ & 0 & 150 & 345 & 495 & 885 & 1045 & 1045 & 1840 & 2195 & 2415 \\
\hline$p(v)$ & -1 & 1 & 2 & 3 & 3 & 4 & 5 & 7 & 8 & 9 \\
\hline
\end{tabular}

The end table (Table 3) shows the shortest routes from point 1 to the given vortices in the graph, and specific routes. The solution of the problem, the shortest route from vortex 1 to vortex 10, illustrating respectively the parking position no. 12 and the point on the runway RWY29, is a minimum route of $2415 \mathrm{~m}$ and route $10 \leftarrow 9 \leftarrow 8 \leftarrow 7 \leftarrow 5 \leftarrow 3 \leftarrow 2 \leftarrow 1$ which corresponds to taxiways M $\rightarrow$ M $3 \rightarrow \mathrm{E} 2 \rightarrow \mathrm{E}$. 


\subsection{Calculation of the Maximum Number of Aircraft for Take- off Operation}

There is a possibility of more than one take-off operation in the $T_{p}$ time interval, so the theoretical maximum number of take-off operations $N_{\max }$ in the "free runway" interval is calculated.

$N_{\max }=\frac{\boldsymbol{W} \boldsymbol{p}-\boldsymbol{T}_{\boldsymbol{s l}}}{\boldsymbol{T}_{\boldsymbol{s s}}}+1$, where $N_{\max } \in \mathbb{Z}^{+} \cup\{\mathbf{0}\}$

Calls of aircraft from $g_{f}$ groups may cause the phenomenon of pushing the aircraft back in such an order that a taxiway conflict is created. TOBT times may be missorted and the separation between take-offs may not be maintained. In order to prevent such phenomena, TOBT time is sorted into groups and separations between operations are set.

In the set $g_{f}=\left\{Z_{1}, Z_{2}, Z_{3}, \ldots, Z_{s}, \ldots, Z_{V}\right\}$ a procedure is performed to sort the target take-off times so that a potential group of aircraft taking off can perform this maneuver safely, with the separation maintained.

$\mathrm{TTOT}_{\mathrm{f}}-1 \leq \mathrm{TTOT}_{\mathrm{f}}+\mathrm{T}_{\mathrm{ss}} \leq \mathrm{TTOT}_{\mathrm{f}+1}+\mathrm{T}_{\mathrm{ss}} \leq \ldots \leq \mathrm{TTOT}_{\mathrm{V}}+\mathrm{T}_{\mathrm{sl}}$

By adding the delay time $T_{o}$ make the above property happen.

$\mathrm{TTOT}_{\mathrm{f}}-1 \not \mathrm{TTOT}_{\mathrm{f}}+\mathrm{T}_{\mathrm{ss}} \Rightarrow \mathrm{T}_{\mathrm{o}}=\mathrm{TTOT}_{\mathrm{f}-1}-\mathrm{TTOT}_{\mathrm{f}}-\mathrm{T}_{\mathrm{ss}}$

If new $\mathrm{TTOT}_{\mathrm{f}}$ have been created in $g_{f}$ groups it is required to check the last aircraft order for take-off, whether the new last TTOT $_{\mathrm{V}}$ time in the group is within the $T_{p}$ range.

TTOT $_{\mathrm{V}}+\mathrm{T}_{\mathrm{sl}} \leq \mathrm{TLDT}_{\mathrm{l}+1}$

All groups for which the above condition is not met are rejected and not taken into account in subsequent stages of the algorithm. After changing the TTOT times in groups, the TOBT time of the aircraft call is also updated by adding the same delay that was added to $\mathrm{T}_{\mathrm{o}}$, the TTOT time. Calculations of aircraft characteristic times in $g_{f}$ groups for all points on the way of the report $D_{s}\left(x_{p}, x_{k}\right)$. Time at point $x_{a}$ of the reported aircraft $s$ belonging to group $f$, on its route to runway $D_{s}\left(x_{p}, x_{k}\right)$.

$T d_{s f}\left(x_{a}\right)=\operatorname{TOBT}_{f}+\frac{\sum \boldsymbol{D}_{\boldsymbol{f}}\left(x_{\boldsymbol{p}}, \boldsymbol{x}_{\boldsymbol{a}}\right) \operatorname{dis}\left(\boldsymbol{x}_{\boldsymbol{p}}, \boldsymbol{x}_{\boldsymbol{i}}\right)}{\boldsymbol{V}_{\boldsymbol{k}}}$

where:

$D_{f}\left(x_{p}, x_{a}\right) \in D_{s}\left(x_{p}, x_{k}\right)$

For arriving aircraft, times at characteristic points are also calculated.

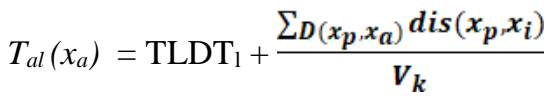


$T_{a l}\left(x_{a}\right)$ - time at point $x_{a}$ of the landing aircraft $l$, on its route from the runway to the parking position $D_{l}\left(x_{p}, x_{k}\right)$.

where:

$D\left(x_{p}, x_{a}\right) \in D_{l}\left(x_{p}, x_{k}\right)$.

Aircraft on the same taxiway may taxi provided that adequate time separation is maintained. Therefore, it is necessary to ensure that in $g_{f}$ groups during taxiing, there is no conflict with a reported aircraft from the group or with a taxiing aircraft that has landed. To prevent such a phenomenon from occurring, dependencies are checked:

$\operatorname{Td}_{(s-1) f}\left(x_{a}\right) \neq T d_{s f}\left(x_{a}\right) \neq T a_{l}\left(x_{a}\right) \neq T a_{l+1}\left(x_{a}\right)$

where:

$x_{a} \in D_{s-1}\left(x_{p}, x_{k}\right) \vee D_{s}\left(x_{p}, x_{k}\right) \vee D_{l}\left(x_{p}, x_{k}\right) \vee D_{l+l}\left(x_{p}, x_{k}\right)$

and

$\operatorname{Td}_{(s-1) f}\left(x_{a}\right), T d_{s f}\left(x_{a}\right), \ldots, T d_{V f}\left(x_{a}\right) \notin\left\langle T a_{l}\left(x_{a}\right)-T_{s k} ; T a_{l}\left(x_{a}\right)+T_{s k}>\right.$

$T d_{(s-1) f}\left(x_{a}\right), T d_{s f}\left(x_{a}\right), \ldots, T d_{V f}\left(x_{a}\right) \notin<T a_{l+1}\left(x_{a}\right)-T_{s k} ; T a_{l+1}\left(x_{a}\right)+T_{s k}>$

where $x_{a} \in D_{s-l}\left(x_{p}, x_{k}\right) \vee D_{s}\left(x_{p}, x_{k}\right) \vee D_{l}\left(x_{p}, x_{k}\right) \vee D_{l+l}\left(x_{p}, x_{k}\right)$

Restriction due to taxiing aircraft that have landed and are taxiing to a parking position. And a limitation due to possible aircraft taxiing to the threshold of the runway:

$$
\begin{aligned}
& T d_{(s-1) f}\left(x_{a}\right) \notin\left\langle T d_{s f}\left(x_{a}\right)-T_{s k} ; T d_{s f}\left(x_{a}\right)+T_{s k}>\right. \\
& T d_{s f}\left(x_{a}\right) \notin\left\langle T d_{(s+1) f}\left(x_{a}\right)-T_{s k} ; T d_{(s+1) f}\left(x_{a}\right)+T_{s k}>\right. \\
& \ldots \\
& T d_{(V-1) f}\left(x_{a}\right) \notin\left\langle T d_{V) f}\left(x_{a}\right)-T_{s k} ; T d_{V) f}\left(x_{a}\right)+T_{s k}>\right.
\end{aligned}
$$

where:

$x_{a} \in D_{s-1}\left(x_{p}, x_{k}\right) \vee D_{s}\left(x_{p}, x_{k}\right) \vee D_{V+1}\left(x_{p}, x_{k}\right) \vee D_{V}\left(x_{p}, x_{k}\right)$

When the above dependency is not met, delay $T_{2}$ is added to time $\operatorname{Td}_{s f}\left(x_{a}\right)$ so that the dependency for all points, calls in a given $g_{f}$ group is fulfilled. The $\mathrm{To}_{2}$ time is added to the conflict aircraft, which is further in the $g_{f}$ group (has a larger $f$ index), and to the other aircraft further in the group.

$$
\begin{gathered}
\operatorname{Td}_{s f}\left(x_{a}\right) \in<T d_{(s+1) f}\left(x_{a}\right)-T_{s k} ; \\
T d_{(s+1) f}\left(x_{a}\right)+T_{s k}>\Longrightarrow T d_{(s+1) f}\left(x_{a}\right)+T o_{2}, T d_{(s+2) f}\left(x_{a}\right)+T o_{2}, \ldots, T d_{V f}\left(x_{a}\right)+T o_{2}
\end{gathered}
$$


After adding the $\mathrm{To}_{2}$ time the taxiing restrictions are checked again. Subsequently, the added $\mathrm{To}_{2}$ delays are also added until the TOBT and TTOT of a given aircraft in $g_{f}$ group are called.

The newly developed TTOT is checked again by the following condition:

\section{TTOTs $\in T_{p}$}

If a call in a newly created group or a group that does not need to be corrected by $\mathrm{To}_{2}$ time, the above dependency is not met, the $g_{f}$ group to which the request belonged is not taken into account in the next stage of the algorithm.

All $g_{f}$ groups meeting the condition create a new set $G_{f i n a l}$.

$G_{\text {final }}=\left\{g_{1}, g_{2}, g_{3}, g_{4}, g_{5}, \ldots, g_{f}\right\}$

where:

$g_{1}, g_{2}, g_{3}, g_{4}, g_{5}, \ldots, g_{f}$ - meets the conditions for taxiing

In $g_{f}$ groups meeting all conditions for taxiing, the sums of priority coefficients are calculated in order to select the most priority groups with the lowest delay in relation to old and new TOBT.

$\operatorname{Pr}\left(g_{f}\right)=\sum_{\boldsymbol{z}_{\boldsymbol{s}}} \boldsymbol{p}_{\boldsymbol{s}}$, for $\quad p_{s} \in Z_{s} \vee Z_{s} \in g_{f}$

In addition, the $\operatorname{Wo}\left(g_{f}\right)$ delay indicator is calculated for each $g_{f}$ group.

$W_{o}\left(g_{f}\right)=\sum_{z_{s}} \boldsymbol{T}_{\boldsymbol{o}}+\boldsymbol{T}_{\boldsymbol{o 2}}+\boldsymbol{T}_{\boldsymbol{o 3}}$, for $\quad T_{o}, T_{o 2}, T_{o 3} \in Z_{s} \bigvee Z_{s} \in g_{f}$

At the current stage of the algorithm, groups that meet taxiing conditions are taken into account and group priorities - $\operatorname{Pr}\left(g_{f}\right)$ and delay indicators - $W_{o}\left(g_{f}\right)$ have been calculated. The group that will be allowed to be pushed back will be the group that meets the conditions that among the $G_{\text {final }}$ group - all calls meeting the taxiing condition, a group or a set of groups is selected:

$\operatorname{Max}\left\{\overline{\overline{\boldsymbol{g}_{\mathbf{1}}}}, \overline{\overline{\boldsymbol{g}_{\mathbf{2}}}}, \overline{\overline{\boldsymbol{g}_{\mathbf{3}}}}, \ldots \overline{\overline{\boldsymbol{g}_{\boldsymbol{f}}}}\right\}=\mathrm{G}_{\mathrm{maxnumb}}$

where:

$\mathrm{g}_{1}, \mathrm{~g}_{2}, \mathrm{~g}_{3}, \mathrm{~g}_{4}, \mathrm{~g}_{5}, \ldots, \mathrm{g}_{\mathrm{f}} \in \mathrm{G}_{\text {final }}$

$\mathrm{G}_{\text {maxnumb }}$ - a set of calls with a maximum number

and

MIN $\left\{\operatorname{Pr}\left(g_{1}\right), \operatorname{Pr}\left(g_{2}\right), \operatorname{Pr}\left(g_{3}\right), \ldots, \operatorname{Pr}\left(g_{f}\right)\right\}=G_{\text {maxnumb/priority }}$

$\mathrm{G}_{\text {maxnumb/priority }}$ - represents a set of calls with a maximum number and minimum priority indicator.

where:

$\mathrm{g}_{1}, \mathrm{~g}_{2}, \mathrm{~g}_{3}, \mathrm{~g}_{4}, \mathrm{~g}_{5}, \ldots, \mathrm{g}_{\mathrm{f}} \in \mathrm{G}_{\text {maxnumb }}$ 
If there is only one group with the above parameters, calls included in these groups are accepted for pushback, with the TOBT time that is dedicated (corrected or not, by the algorithm) to the call. All other calls that have not received permission for pushback, have the delay $T_{o 3}$ added to their times, so that:

$\mathrm{TOBT}_{\mathrm{s}}+\mathrm{To}_{3} \in<\mathrm{TLDT}_{\mathrm{l}+1}+\mathrm{T}\left(\mathrm{b}_{\mathrm{d}}\right) ; \mathrm{TLDT}_{\mathrm{l}+2}-\mathrm{T}_{\mathrm{sl}}>$

for $b_{d}=b_{l}, b_{l} \in Z_{l+1}$

When there is more than one group, after checking the above dependencies, the group is selected which:

$\operatorname{MIN}\left\{\mathrm{Wo}\left(\mathrm{g}_{1}\right), \mathrm{Wo}\left(\mathrm{g}_{2}\right), \mathrm{Wo}\left(\mathrm{g}_{3}\right), \mathrm{Wo}\left(\mathrm{g}_{4}\right), \ldots, \mathrm{Wo}\left(\mathrm{g}_{\mathrm{f}}\right)\right\}=\mathrm{g}_{\mathrm{wyp}}$

where:

$\mathrm{g}_{1}, \mathrm{~g}_{2}, \mathrm{~g}_{3}, \mathrm{~g}_{4}, \mathrm{~g}_{5}, \ldots, \mathrm{g}_{\mathrm{f}} \in \mathrm{G}_{\text {maxlicz/priority }}$

One group with the above parameters is accepted for pushback, with the TOBT time that is dedicated (corrected or not, by the algorithm) to the call. All other calls that have not received permission for pushback, have the delay $T_{o 3}$ added to their times, so that:

$\mathrm{TOBT}_{\mathrm{s}}+\mathrm{To}_{3} \in<\mathrm{TLDT}_{\mathrm{l}+1}+\mathrm{T}\left(\mathrm{b}_{\mathrm{d}}\right) ; \mathrm{TLDT}_{\mathrm{l}+2}-\mathrm{T}_{\mathrm{sl}}>$

for $b_{d}=b_{l+1}, b_{l+1} \in Z_{l+1}$

After allowing a given group to be pushed back, the algorithm is resumed for subsequent air operations, from the beginning, but the existing aircraft calls retain their priority value.

\section{Conclusions}

The proposed algorithm for the aircraft pushback sequence, limits the amount of exhaust emissions into the atmosphere, because it assumes that the selected route of an aircraft, from the parking position to the runway, is the shortest and the movement of the aircraft is without unnecessary stops, waiting time and the delays associated with the movement of other aircraft on the maneuver area. The main purpose of the algorithm is to increase the capacity of the maneuvering area, by improving the capacity factor specified in the Official Journal of the European Union (the difference between the ordered time and the actual take-off).

The proposed aircraft departure system can provide a minimum taxiway from the parking position to the runway. The conditions imposed on the aircraft call groups, guarantee the smooth execution of operations and the absence of disturbances to the other traffic participants, in the maneuvering area. Selecting the maximum group ensures the maximum operational use of the slot.

The system completely eliminates the queuing phenomenon, because the inclusion of an aircraft in traffic is only possible, if there is a possibility to perform take-off 
or deicing operations, at the designated time of arrival, of the aircraft. Thanks to the exchange of information in real time, the system can react dynamically to the traffic situation in the maneuvering area and significantly improve the operational preparation of the individual partners, involved in the aircraft service. One of the effects of the departure system is the relief of the air traffic controller.

The air traffic controller, having the information provided by the system, receives hints concerning the minimum taxiing route for the aircraft, which reduces the activities performed and analysis made before giving permission to taxiing, takeoff or start the aircraft engines.

From the analyzed times for leaving the blocks, it can be seen that most of the aircraft, that received permission for pushback to the taxiway, received them after a scheduled time. The delay resulting from the late pushback of an aircraft, to the taxiway, accounts for, as much as, $62 \%$ of all delays. This delay is due to the aircraft's readiness to taxi. Many operations can contribute to the delay, as before pushback, an aircraft is handled by a handling agent, who loads the baggage, boards the passengers and pushes back the aircraft itself. An aircraft is also checked by its Captain, before each take-off and refueling the aircraft is also possible.

All the above mentioned operations can be shortened with improved operational preparation, resulting from the information provided herein. A properly functioning information exchange platform, described in A-CDM makes it possible to perform the operations, preceding the aircraft pushback to the taxiway, in a way, that minimizes delays.

\section{References}

[1] SESAR Consortium, "European ATM Master Plan - Edition 2," Roadmap Sustain. Air Traffic Manag., 2012, doi: doi:10.2829/512525

[2] P. O. Box, “A-CDM documentation ZURICH AIRPORT,” pp. 1-26

[3] D. Karapetyan, J. A. D. Atkin, A. J. Parkes, and J. Castro-Gutierrez, "Lessons from building an automated pre-departure sequencer for airports," Ann. Oper. Res., Vol. 252, No. 2, pp. 435-453, 2017, doi: 10.1007/s10479015-1960-Z

[4] A. Kwasiborska, "Sequencing landing aircraft process to minimize schedule length," in Transportation Research Procedia, 2017, Vol. 28, doi: 10.1016/j.trpro.2017.12.175

[5] D. Bãhme, R. Brucherseifer, and L. Christoffels, "Coordinated arrival departure management," in Proceedings of the $7^{\text {th }}$ USA/Europe Air Traffic Management Research and Development Seminar, ATM 2007, 2007

[6] G. Pavese, M. Bruglieri, A. Rolando, and R. Careri, "DMAN-SMANAMAN OPTIMIZATION AT LINATE AIRPORT," 2017 
[7] A. Lieder, D. Briskorn, and R. Stolletz, "A dynamic programming approach for the aircraft landing problem with aircraft classes," Eur. J. Oper. Res., 2015, doi: 10.1016/j.ejor.2014.11.027

[8] D. Jagieło and A. Kwasiborska, The concept of tool to support the work of air traffic controller in the field of aircraft landing scheduling in the TMA with little traffic, Vol. 715. 2017

[9] International Civil Aviation Organization, Doc 4444 - Air Traffic Management - Procedures for Air Navigation Services. 2016

[10] A. Kwasiborska and J. Skorupski, "Analysis of the process of merging air traffic streams. Case study of TMA Warsaw," in Communications in Computer and Information Science, 2018, Vol. 897, pp. 320-334, doi: 10.1007/978-3-319-97955-7_22

[11] Á. Kovács, E. Németh, and K. M. Hangos, "Modeling and optimization of runway traffic flow using coloured Petri nets," in Proceedings of the $5^{\text {th }}$ International Conference on Control and Automation, ICCA'05, 2005, doi: 10.1109/icca.2005.1528246

[12] A. Kwasiborska and A. Stelmach, "Analysis of airport traffic in the context of environmental throughput," Transp. Probl., Vol. 9, No. 1, 2014

[13] E. L. Lawler, J. K. Lenstra, A. H. G. Rinnooy Kan, and D. B. Shmoys, "Sequencing and scheduling: Algorithms and complexity," Handbooks in Operations Research and Management Science. 1993, doi: 10.1016/S09270507(05)80189-6

[14] N. Manager, "ATFCM users manual,” 2018

[15] ICAO, Continuous Descent Operations (CDO) Manual. 2010

[16] S. Caprì and M. Ignaccolo, "Genetic algorithms for solving the aircraftsequencing problem: The introduction of departures into the dynamic model,” J. Air Transp. Manag., 2004, doi: 10.1016/j.jairtraman.2004.05.004

[17] L. Mantecchini, M. N. Postorino, and E. Gualandi, "Integration between aircraft and handling vehicles during taxiing procedures to improve airport sustainability Integration between aircraft and handling vehicles during taxiing procedures to improve airport sustainability," no. May, 2016, doi: 10.2495/TDI-V1-N1-28-42

[18] M. Zhang, Q. Huang, S. Liu, and H. Li, "Multi-Objective Optimization of Aircraft Taxiing on the Airport Surface with Consideration to Taxiing Conflicts and the Airport Environment," pp. 1-27, 2019

[19] N. Per and A. A. L. Volo, "Point Merge System Results of ENAV Prototyping Sessions," 2008

[20] S. Padr, "A multi-objective optimization approach to the ground handling scheduling problem Silvia Padr ' on Astorga," no. May, 2014 
[21] A. Javaid and E. Engineers, "Understanding Dijkstra 's Algorithm Abstract : Introduction:," no. January 2013, 2018, doi: $10.2139 /$ ssrn.2340905

[22] T. Xiaowei, "Research on Aircraft Pushback Procedure in Busy Airport", 2010 International Conference on Optoelectronics and Image Processing, Haikou, 2010, pp. 201-204, doi: 10.1109/ICOIP.2010.131

[23] https://www.hackerearth.com/practice/algorithms/graphs/shortest-pathalgorithms/tutorial/

[24] https://www.aviation.govt.nz/licensing-and-certification/pilots/flighttraining/flight-instructor-guide/taxiing/

[25] https://www.geeksforgeeks.org/dijkstras-shortest-path-algorithm-greedyalgo-7/

[26] L. Banachowski, K. Diks, W. Rytter, “Algorytmy i struktury danych”, Wydawnictwa Naukowo - Techniczne, 2006 